\title{
A STUDY OF WELL-BALANCED FINITE VOLUME METHODS AND REFINEMENT INDICATORS FOR THE SHALLOW WATER EQUATIONS
}

\author{
SUDI MUNGKASI
}

\begin{abstract}
2010 Mathematics subject classification: primary 65M08, 65N08; secondary 76B15, 76M12.
Keywords and phrases: finite volume methods, dam break, debris avalanche, Carrier-Greenspan solution, numerical entropy production, weak local residuals, adaptive mesh refinement, shallow water equations.
\end{abstract}

Water flows can be modelled mathematically and one available model is the shallow water equations. This thesis studies solutions to the shallow water equations analytically and numerically. The study is divided into three parts.

The first part is about well-balanced finite volume methods to solve steady and unsteady state problems. A method is said to be well balanced if it preserves an unperturbed steady state at the discrete level [10]. We implement hydrostatic reconstructions proposed by Audusse et al. [1] for the well-balanced methods with respect to the steady state of a lake at rest. Four combinations of quantity reconstructions are tested. Our results indicate an appropriate combination of quantity reconstructions for dealing with steady and unsteady state problems [3].

The second part presents some new analytical solutions to debris avalanche problems [5, 7] and reviews the implicit Carrier-Greenspan periodic solution for flows on a sloping beach [8]. The analytical solutions to debris avalanche problems are derived using characteristics and a variable transformation technique. The analytical solutions are used as benchmarks to test the performance of numerical solutions. For the Carrier-Greenspan periodic solution, we show that the linear approximation of the Carrier-Greenspan periodic solution may result in large errors in some cases. If an explicit approximation of the Carrier-Greenspan periodic solution is needed, higher order approximations should be considered. We propose second order approximations of the Carrier-Greenspan periodic solution and present a way to get higher order approximations.

The third part discusses refinement indicators used in adaptive finite volume methods to detect smooth and nonsmooth regions. In the adaptive finite volume methods, smooth regions are coarsened to reduce the computation costs and nonsmooth regions are refined to get more accurate solutions. We consider

Thesis submitted to The Australian National University, September 2012. Degree approved, February 2013. Supervisor: Associate Professor Stephen Gwyn Roberts.

(c) 2013 Australian Mathematical Publishing Association Inc. 0004-9727/2013 \$16.00 
the numerical entropy production [11] and weak local residuals [2] as refinement indicators. Regarding the numerical entropy production, our work is the first to implement the numerical entropy production as a refinement indicator into adaptive finite volume methods used to solve the shallow water equations. Regarding weak local residuals, we propose formulations to compute weak local residuals on nonuniform meshes. Our numerical experiments show that both the numerical entropy production and weak local residuals are successful as refinement indicators.

Some publications corresponding to this thesis are listed in [3-9].

\title{
References
}

[1] E. Audusse, F. Bouchut, M. O. Bristeau, R. Klein and B. Perthame, 'A fast and stable well-balanced scheme with hydrostatic reconstruction for shallow water flows', SIAM J. Sci. Comput. 25 (2004), 2050-2065.

[2] S. Karni, A. Kurganov and G. Petrova, 'A smoothness indicator for adaptive algorithms for hyperbolic systems', J. Comput. Phys. 178 (2002), 323-341.

[3] S. Mungkasi and S. G. Roberts, 'On the best quantity reconstructions for a well balanced finite volume method used to solve the shallow water wave equations with a wet/dry interface', ANZIAM J. (Electronic Supplement) 51 (2010), C48-C65.

[4] S. Mungkasi and S. G. Roberts, 'A finite volume method for shallow water flows on triangular computational grids'. Proceedings of IEEE International Conference on Advanced Computer Science and Information System (ICACSIS) (Faculty of Computer Science, The University of Indonesia, Jakarta, 2011), 79-84.

[5] S. Mungkasi and S. G. Roberts, 'A new analytical solution for testing debris avalanche numerical models', ANZIAM J. (Electronic Supplement) 52 (2011), C349-C363.

[6] S. Mungkasi and S. G. Roberts, 'Numerical entropy production for shallow water flows', ANZIAM J. (Electronic Supplement) 52 (2011), C1-C17.

[7] S. Mungkasi and S. G. Roberts, 'Analytical solutions involving shock waves for testing debris avalanche numerical models', Pure Appl. Geophys. 169 (2012), 1847-1858.

[8] S. Mungkasi and S. G. Roberts, 'Approximations of the Carrier-Greenspan periodic solution to the shallow water wave equations for flows on a sloping beach', Internat. J. Numer. Methods Fluids 69 (2012), 763-780.

[9] S. Mungkasi and S. G. Roberts, 'Behaviour of the numerical entropy production of the oneand-a-half-dimensional shallow water equations', ANZIAM J. (Electronic Supplement) 54 (2013), C18-C33.

[10] S. Noelle, N. Pankratz, G. Puppo and J. R. Natvig, 'Well-balanced finite volume schemes of arbitrary order of accuracy for shallow water flows', J. Comput. Phys. 213 (2006), 474-499.

[11] G. Puppo, 'Numerical entropy production for central schemes', SIAM J. Sci. Comput. 25 (2004), 1382-1415.

\author{
SUDI MUNGKASI, Department of Mathematics, Sanata Dharma University, \\ Mrican, Tromol Pos 29, Yogyakarta 55002, Indonesia \\ e-mail: sudi@usd.ac.id \\ and \\ Mathematical Sciences Institute, The Australian National University, \\ Canberra, ACT 0200, Australia \\ e-mail: sudi.mungkasi@anu.edu.au
}

